\title{
Selenium-Containing Amino Acids Protect Dextran Sulfate Sodium-Induced Colitis via Ameliorating Oxidative Stress and Intestinal Inflammation
}

This article was published in the following Dove Press journal:

Journal of Inflammation Research

\author{
Chengxin $\mathrm{Shi}^{1}{ }^{1} *$ \\ Fengli Yue ${ }^{2, *}$ \\ Feiyu Shi ${ }^{\prime}$ \\ Qian Qin' \\ Lizhao Wang $\mathbb{D}^{\prime}$ \\ Guanghui Wang' \\ Lijun $\mathrm{Mu}^{\prime}$ \\ Dan Liu' \\ Yaguang $\mathrm{Li}^{\prime}$ \\ Tianyu $\mathrm{Yu}^{\prime}$ \\ Junjun She $\mathbb{D}^{\prime}$ \\ 'Department of General Surgery, The \\ First Affiliated Hospital of Xi'an Jiaotong \\ University, Xi'an, Shaanxi 7I006I, \\ People's Republic of China; ${ }^{2}$ College of \\ Basic Medical Sciences, Jilin University, \\ Changchun I3002I, People's Republic of \\ China
}

*These authors contributed equally to this work

\begin{abstract}
Background: Inflammatory bowel disease (IBD) is characterized by chronic relapsing inflammation of the gastrointestinal tract. Oxidative stress plays a pivotal role in the pathogenesis of IBD. Selenium-containing amino acids reportedly have anti-oxidative and anti-inflammatory properties, but it remains unknown if selenium-containing amino acids can be used to treat IBD. This study aimed to investigate the effects of two selenium-containing amino acids - selenocysteine and selenocystine - on oxidative stress and chronic inflammation in a mouse model of dextran sulfate sodium (DSS)-induced IBD.
\end{abstract}

Methodology: C57BL/6 mice were randomly assigned to the following six groups: control, DSS, DSS+selenocysteine, DSS+selenocystine, DSS+sodium selenite, and DSS $+\mathrm{N}$-acetylcysteine (NAC). IBD was induced by $3 \%$ DSS. Pro-inflammatory cytokines [interleukin-1 $\beta$ (IL-1 $\beta$ ), monocyte chemotactic protein 1 (MCP-1), IL-6, and tumor necrosis factor- $\alpha(\mathrm{TNF}-\alpha)$ ] and markers for oxidative and anti-oxidative stress [malondialdehyde (MDA), reactive oxygen species (ROS), superoxide dismutase (SOD), and glutathione peroxidase $(\mathrm{GPx})$ ] were measured using immunohistochemical analysis.

Results: Selenocysteine and selenocystine significantly attenuated IBD-related symptoms, including preventing weight loss, decreasing disease activity index (DAI) scores, and increasing colon length. Selenocysteine and selenocystine significantly ameliorated the DSSinduced oxidative stress, as demonstrated by a reduction in ROS and MDA activity and an increase in SOD and GPx activity. IL-1, MCP-1, IL-6, and TNF- $\alpha$ levels were significantly increased in the IBD mice, while treatment with the selenium-containing amino acids significantly reduced the levels of these pro-inflammatory cytokines. In vivo safety analysis showed minimal side effects of the selenium-containing amino acids.

Conclusion: We found that selenocysteine and selenocystine ameliorated DSS-induced IBD via reducing oxidative stress and intestinal inflammation, indicating that selenium-containing amino acids could be a novel therapeutic option for patients with IBD.

Keywords: Inflammatory bowel diseases, colitis, selenium-containing amino acids, selenocysteine, selenocystine, oxidative stress, inflammation

\section{Introduction}

Inflammatory bowel disease (IBD), including ulcerative colitis and Crohn's disease, is characterized by chronic relapsing and persistent inflammation of the intestinal mucosa. ${ }^{1,2}$ Although the pathogenesis of IBD is not fully understood, intestinal barrier dysfunction, oxidative stress, inflammation, and microbiota-host interaction play key
Department of General Surgery, The First Affiliated Hospital of Xi'an Jiaotong University, No. 277, Yanta West Road, Xi'an, Shaanxi 71006I, People's Republic of China Tel +86-I7765856985

Email shejunjun@mail.xjtu.edu.cn
Journal of Inflammation Research 2021:14 85-95 
roles in the development of IBD. ${ }^{3,4}$ At present, the main pharmacological treatment options for IBD patients include aminosalicylic acid, glucocorticoids, antibiotics, immunosuppressants, and biological agents. ${ }^{5}$ These drugs exert therapeutic effects in patients with IBD through regulating inflammation in the microenvironment, but long-term use of these drugs may cause side effects and increase risk of developing other diseases. ${ }^{6}$ As such, new treatments for IBD are needed.

It has long been recognized that oxidative stress is critical in the pathogenesis and progression of IBD. For instance, reactive oxygen species (ROS) have been proposed to contribute to the development of IBD, especially since oxidative stress can promote the pro-inflammatory process by activating several immune cells. ${ }^{7}$ Previous findings have implicated anti-oxidants as potential new treatments for IBD, such as synthetic active pharmaceutical ingredients (API), natural compounds, ions, and vitamins. ${ }^{8,9}$ Therefore, anti-oxidative drugs could potentially improve IBD.

Selenium is a well-known essential trace element, which exists mainly as selenocysteine containing selenoproteins in the human body. ${ }^{10,11}$ Selenoprotein family members play important roles in maintaining the healthy function of the immune system. ${ }^{12,13}$ Previous studies have demonstrated that both serum selenoprotein P levels and glutathione peroxidase activity are decreased in patients suffering from Crohn's disease, ${ }^{14}$ and that abnormally low selenium levels are associated with elevated levels of pro-inflammatory mediators [eg prostaglandin E2 (PGE2), interleukin-6 (IL6 ), and tumor necrosis factor- $\alpha(\mathrm{TNF}-\alpha)]$ in patients with IBD. ${ }^{15}$ Sodium selenite, an inorganic selenium supplement, has been demonstrated to exert a protective role in attenuating dextran sulfate sodium (DSS)-induced colitis in mice. ${ }^{16}$ Similar to sodium selenium, organic selenium supplements exhibit higher bioactivity and lower toxicity. ${ }^{17}$ Several previous studies have reported that organic selenium-containing amino acids (eg selenocysteine and selenocystine) act as both direct anti-oxidants and a source of selenium in the synthesis of selenium-dependent proteins. ${ }^{18,19}$ However, it remains unknown whether and how selenium-containing amino acids exert beneficial impacts in IBD. In this study, we tested the hypothesis that selenocysteine and selenocystine can be used to effectively prevent or treat DSS-induced IBD.

We aimed to investigate the effects of two seleniumcontaining amino acids - selenocysteine and selenocystine - on oxidative stress and chronic inflammation in a mouse model of DSS-induced IBD, and compared their activity with sodium selenite and N-acetylcysteine (NAC).

\section{Materials and Methods \\ Reagents}

Selenocysteine and selenocystine (Figure 1), sodium selenite, and NAC were purchased from Sigma Aldrich (St. Louis, MO, USA). DSS was obtained from MP Biomedicals (36000-50000 MW) (Santa Ana, CA, USA).

\section{Experimental Animals}

Male C57BL/6 mice at 8 weeks of age were purchased from Beijing Vital River Laboratory Animal Technology (Beijing, China). The mice were maintained in the animal facility at the College of Basic Medical Sciences, Xi'an Jiaotong University (Xi'an, Shanxi, China). All mice were acclimatized for at least 7 days prior to experimentation. All animal protocols were reviewed and approved by the Ethics Committee for the Use of Experimental Animals of Xi' an Jiaotong University (Xi' an, Shanxi, China), and the Committee for the Purpose of Control and Supervision of Experimentation on Animals (CPCSEA) guidelines were followed during the experiment.

\section{IBD Induction and Treatment}

C57BL/6 mice were randomly allocated to the following six groups: control, DSS, DSS+selenocysteine, DSS+selenocystine, DSS+sodium selenite, and DSS+NAC. IBD
A<smiles>NC(C[Se][Se]CC(N)C(=O)O)C(=O)O</smiles>

B

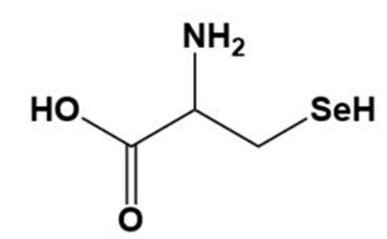

Figure I Chemical structure of selenium-containing amino acids used in this study. Chemical structure of $(\mathbf{A})$ selenocystine and $(\mathbf{B})$ selenocysteine. 
was induced by 3\% (wt./vol) DSS (dissolved in drinking water and administered ad libitum). DSS-challenged mice were treated with selenocysteine, selenocystine, or sodium selenite with the same selenium concentrations $(0.9 \mathrm{mg} /$ $\mathrm{kg}$ ) or NAC (2 mg/kg) [dissolved in phosphate-buffered saline (PBS) and administered vial oral gavage]. Mice in the control group were treated with the same volume of PBS.

\section{Disease Activity Index (DAI)}

DAI scores were calculated as the sum of the scores of stool consistency [hard (0), soft (2), and diarrhea (4)], fecal occult blood [negative (0), positive (2), macroscopic (4)], and weight loss $[<1 \%(0), 1-5 \%$ (1), 5-10\% (2), $10-20 \%$ (3), and $>20 \%$ (4)].

\section{Histological Examinations}

Upon completion of the proposed experiments, the mice were sacrificed, after which the entire colon of each mouse was removed. The colon tissues were soaked in $4 \%$ paraformaldehyde overnight and subsequently sent to the pathological core facility of the Xi'an Jiaotong University for histological analysis. In brief, the paraffinembedded colon sections were stained with hematoxylin and eosin $(\mathrm{H} \& \mathrm{E})$, visualized under a microscope, and reviewed by independent pathologists. Severity of inflammation was assessed using histology scores as follows: no inflammation (0), slight inflammation (1), moderate inflammation (2), and severe inflammation (3); depth of injury: none (0), mucosa (1), mucosa and submucosa (2), and transmural (3); PMN infiltration/HPF: $(0,<5$; 1, 5-20; 2 , 21-60; 3, 61-100; 4, >100); crypt damage: none (0), basal 1/3 (1), basal 2/3 (2), only surface epithelium intact (3), entire crypt lost (4), and adjusted to tissue involvement by multiplying the percentage factor (x1: $0-25 \%, \mathrm{x} 2$ : 26-50\%, x3: 51-75\%, x4: 76-100\%).

\section{Examination of Oxidative and}

\section{Anti-Oxidative Stress Markers in Colon Tissues}

Oxidative and anti-oxidative stress markers in the colon tissues, including ROS, malondialdehyde (MDA), superoxide dismutase (SOD), and glutathione peroxidase (GPx), were measured using ROS, MDA, SOD, and GPx assay kits (Nanjing Jiancheng Bioengineering Institute, Nanjing, Jiangsu, China) according to the manufacturer's instructions.

\section{Determination of Pro-Inflammatory Cytokines IL-I, MCP-I, IL-6, and TNF- $\alpha$ in Colon Tissues}

A number of important pro-inflammatory cytokines, including IL-1, MCP-1, IL-6, and TNF- $\alpha$, were measured in colon tissues using an MCYTOMAG-70K Kit (Merck, Germany) following the manufacturer's instructions.

\section{In vivo Safety Profiles}

After 7 days of treatment, the experimental mice were sacrificed. The heart, liver, lung, kidney, and spleen were removed, fixed, and stained with H\&E. Biochemical tests, including alanine aminotransferase (ALT), aspartate aminotransferase (AST), urea nitrogen (BUN), and creatinine (CRE), were analyzed automatically using Coulter LX2D instrumentation (Beckman, Brea, CA).

\section{Statistical Analysis}

Statistical analyses were carried out using GraphPad Prism software (La Jolla, CA). Data are expressed as means \pm standard errors of mean (SEM). The student's $t$ test was performed for a simple two-sample comparison to determine the significance of differences between groups. For comparison of multiple samples, one-way analysis of variance (ANOVA) with Tukey's post-hoc test was conducted to analyze differences between groups.

\section{Results}

\section{Selenium-Containing Amino Acids Attenuate Symptoms of IBD in Mice}

We first examined the effects of selenium-containing amino acids on the characteristic symptoms of IBD (eg weight loss, diarrhea, and rectal bleeding), pro-inflammatory response, and intestinal histological alterations in mice following the DSS challenge. As shown in Figure 2, the DSSchallenged group had significant decreases in body weight (Figure 2A), increases in DAI scores (Figure 2B), and shortened colon lengths (Figure 2C and D) compared to the control group. Notably, selenocysteine and selenocystine treatment significantly increased body weight, reduced the DAI score, and prevented DSS-induced shortening of colon length, suggesting that selenium-containing amino acids can attenuate the main symptoms of IBD in mice. The selenium-containing amino acids had greater effects on relieving the main symptoms of IBD compared to sodium selenite and NAC alone. 
A

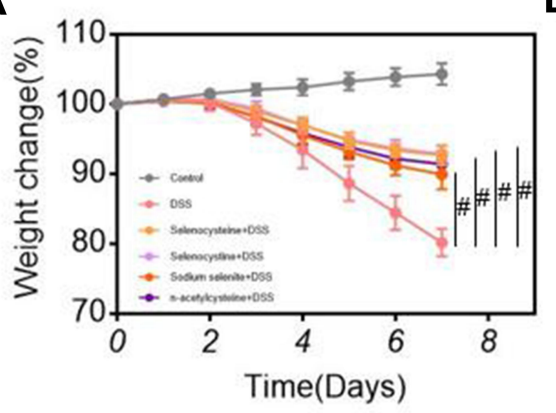

D

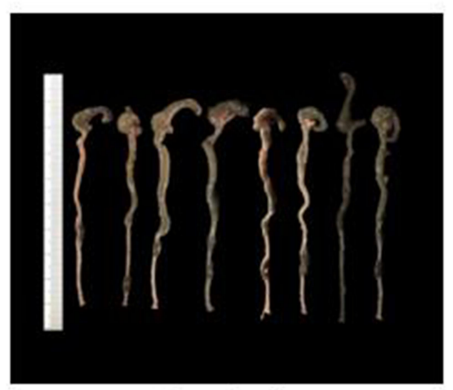

Control

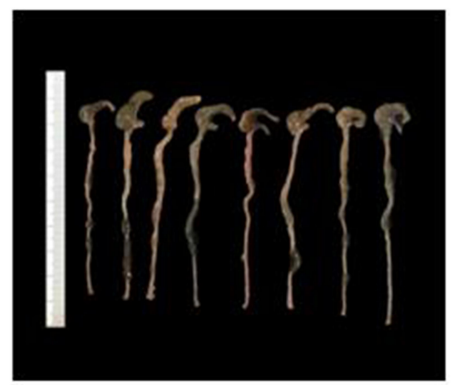

Selenocystine+DSS
B

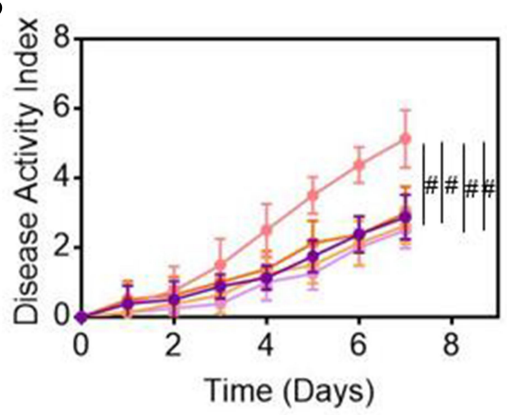

C

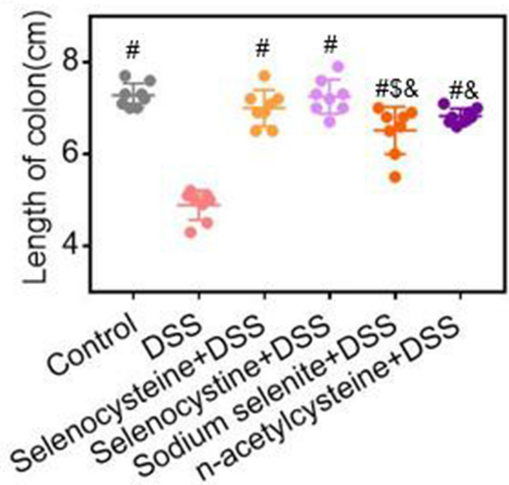

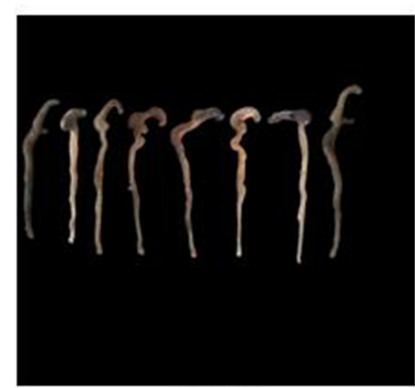

DSS

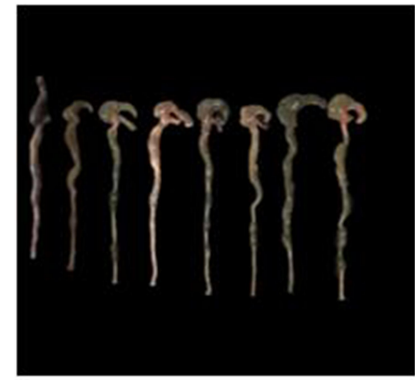

Sodium selenite+DSS

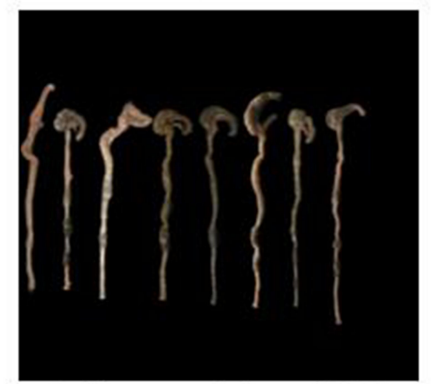

Selenocysteine+DSS

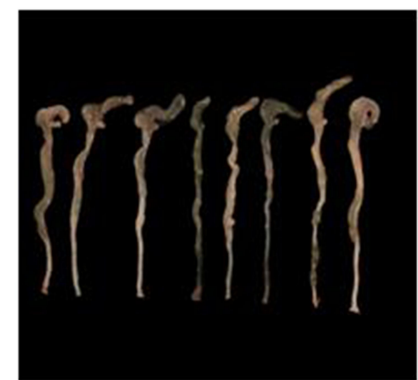

n-acetylcysteine+DSS

Figure 2 Effects of selenium-containing amino acids on the main symptoms of IBD in mice. Weight loss, DAl scores, and colon length were compared between groups. (A) Weight loss; (B) DAl scores; (C) colon length; and (D) colon images in different groups. Eight mice per group; ${ }^{\#} \mathrm{P}<0.05$, compared with DSS group. ${ }^{\$} \mathrm{P}<0.05$, compared with selenocysteine group. ${ }^{\&} \mathrm{P}<0.05$, compared with selenocystine group. Data are expressed as mean \pm SEM.

\section{Selenium-Containing Amino Acids} Ameliorate Inflammatory Injury in

\section{a Mouse Model of IBD}

Histopathological examinations were performed to evaluate the effects of selenium-containing amino acids on inflammatory injury in the mouse model of IBD. As shown in Figure 3, the DSS-challenged group had severe inflammation, abnormal alterations in epithelial structure, mucosal lesions, increased leucocyte infiltration in the mucosa and submucosa, vascularization, edema, and loss of crypts compared to the control group. However, the DSS-induced abnormalities of the epithelial cell lining, muscle layer thickness, and infiltration of immune cells were significantly less in the mice treated with selenocysteine and selenocystine compared to the other groups. Expectedly, the pathological scores of the mice treated with selenium-containing amino acids were significantly lower compared to the DSS-challenged mice (Figure 3B). Consistent with the DAI scores, the pathological scores of mice treated with sodium selenite or NAC were significantly higher than the scores in the selenocysteine or selenocystine group.

\section{Selenium-Containing Amino Acids \\ Reduce Oxidative Stress in the DSS-Induced Mouse Model of IBD}

To evaluate the effects of selenium-containing amino acids on oxidative stress in the DSS-induced mouse model of IBD, we measured levels of oxidative stress markers, including ROS, MDA, and SOD activity, in the colon tissues. As shown in 
A

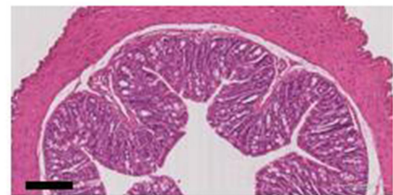

Selenocysteine+DSS

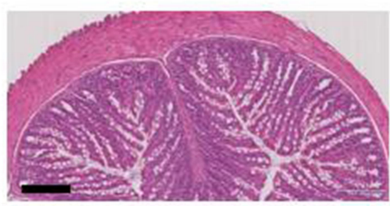

Control

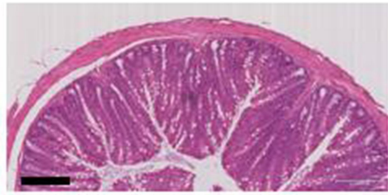

Selenocystine+DSS

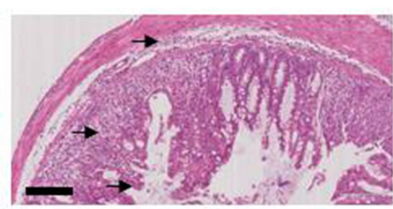

DSS

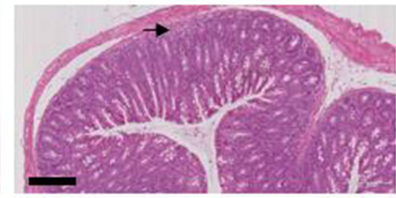

Sodium selenite+DSS

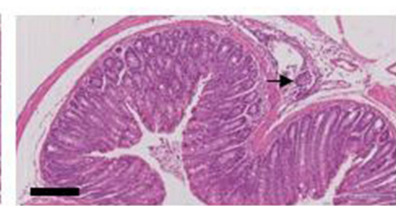

n-acetylcysteine+DSS

B

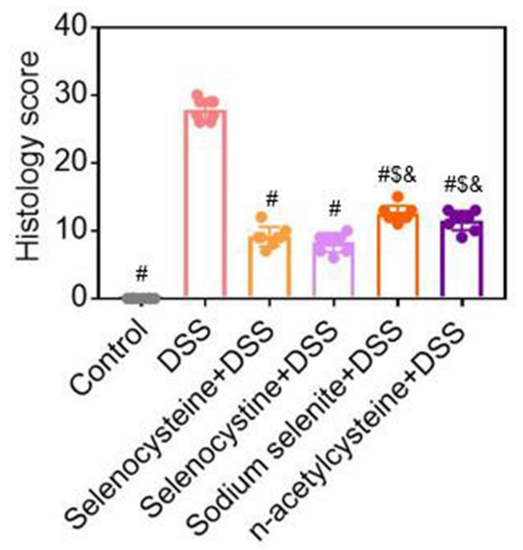

Figure 3 Histological examinations of effects of selenium-containing amino acids on DSS-induced IBD in mice. (A) Representative H\&E-stained colon sections of each group (scale bar, $200 \mu \mathrm{m}$ ); (B) Histological scoring of mice treated with selenium-containing amino acids in DSS-induced IBD. Eight mice per group; ${ }^{P} \mathrm{P}<0.05$, compared with DSS group. ${ }^{\$} P<0.05$, compared with selenocysteine group. ${ }^{\&} P<0.05$, compared with selenocystine group. Data are expressed as mean \pm SEM.

Figure $4 \mathrm{~A}-\mathrm{C}$, mice in the DSS-challenged group showed significantly higher ROS and MDA levels, but lower SOD activity, compared to the control mice. As expected, selenocysteine and selenocystine treatment significantly reduced the MDA and ROS levels and increased the SOD activity. Interestingly, NAC treatment significantly increased the SOD activity, while sodium selenite did not alter SOD activity. To determine the relationship between the anti-oxidant capability of selenium-containing amino acids and their colonic protective effect, we measured GPx activity in the colon tissues. As shown in Figure 4D, DSS challenge was associated with a significant decrease in GPx activity, whereas treatment with selenocysteine or selenocystine significantly restored the GPx activity in the DSS-challenged mice.

\section{Selenium-Containing Amino Acids Reduce Levels of Pro-Inflammatory Cytokines in a Mouse Model of IBD}

To evaluate the effects of selenium-containing amino acids on inflammation, we measured levels of IL-1, MCP-1, IL-
6 , and TNF- $\alpha$ in the colon tissues using ELISA. As shown in Figure 5, pro-inflammatory cytokine levels in the DSSchallenged mice were significantly higher compared to the levels in the control mice. Selenocysteine and selenocystine treatment decreased the levels of all colonic proinflammatory cytokines. Similar effects were observed in the sodium selenite and NAC groups.

\section{In vivo Safety Profiling of Selenium-Containing Amino Acids Shows Minimal Side Effects}

In vivo safety profiling of selenium-containing amino acids was evaluated using biochemical tests and H\&E staining of the heart, liver, spleen, lung, and kidney. As shown in Figure $6 \mathrm{~A}-\mathrm{D}$, negligible pathological changes were found in terms of serum biochemical parameters, including AST, ALT, BUN, and CRE, in all of the groups. There were no histopathologic alterations in the heart, liver, spleen, lung, and kidney tissues in the treatment groups (Figure 7), suggesting 
A

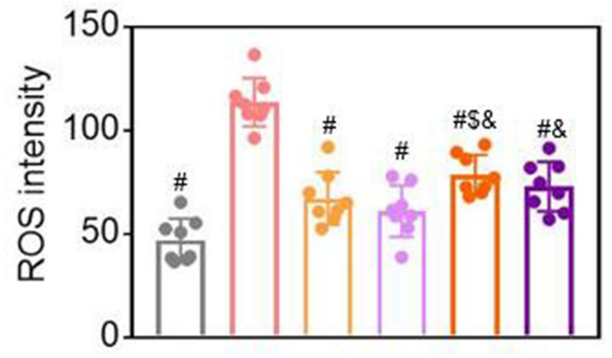

C

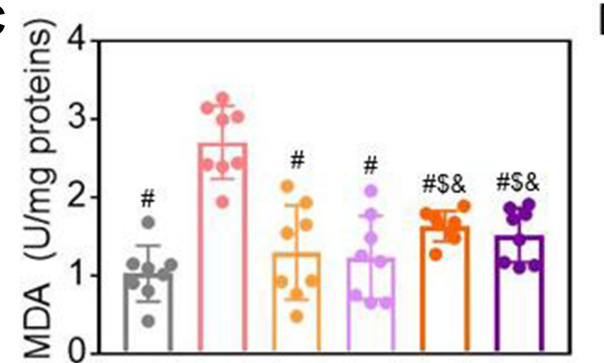

B

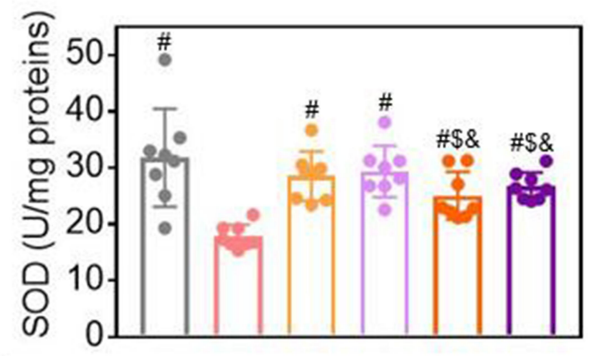

$\mathrm{D}$

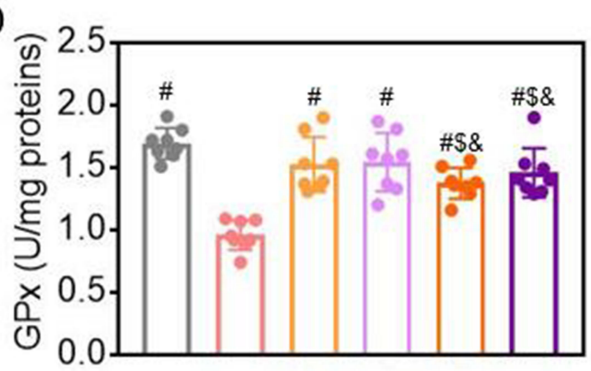

\section{Control DSS Selenocysteine+DSS Selenocystine+DSS \\ Sodium selenite+DSS \\ n-acetylcysteine+DSS}

Figure 4 Effects of selenium-containing amino acids on oxidative stress markers in DSS-induced IBD in mice. Levels of oxidative stress markers including (A) ROS, (B) SOD, (C) MDA, and (D) GPx in colon tissues from different groups. Eight mice per group; ${ }^{\# P}<0.05$, compared with DSS group. ${ }^{\$} \mathrm{P}<0.05$, compared with selenocysteine group. ${ }^{\&} P<0.05$, compared with selenocystine group. Data are expressed as mean \pm SEM.

\section{A}
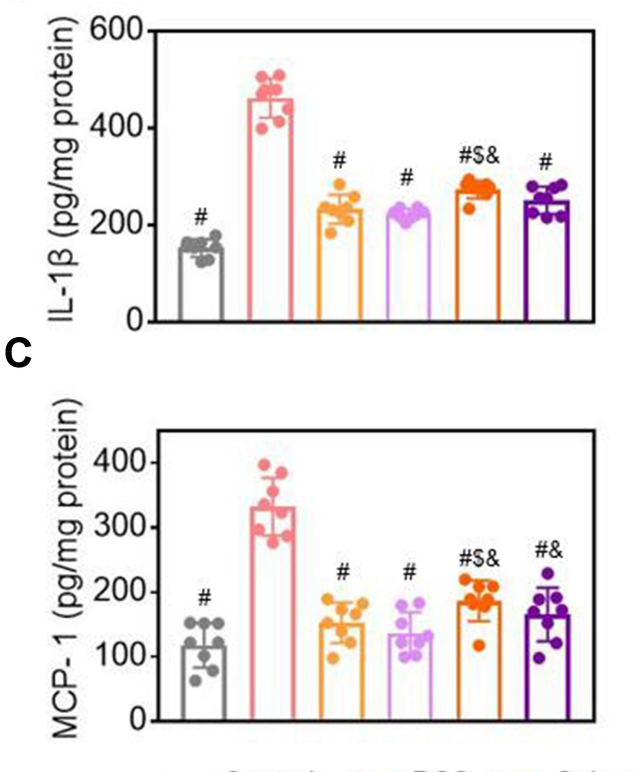

B

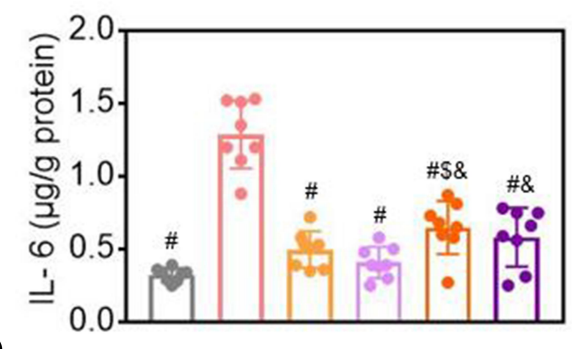

D

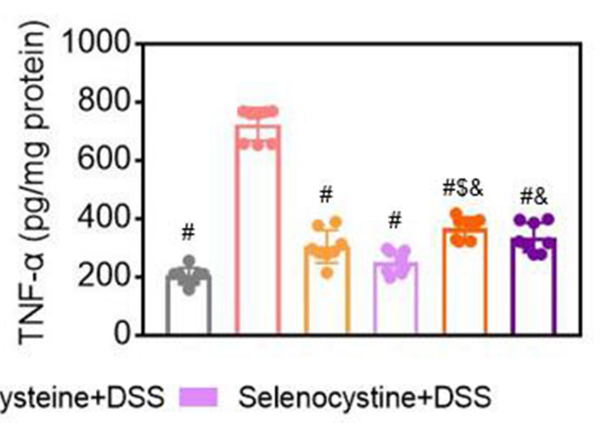

- Sodium selenite+DSS

n-acetylcysteine+DSS

Figure 5 Effects of selenium-containing amino acids on levels of pro-inflammatory cytokines in DSS-induced IBD in mice. Levels of (A) IL-I $\beta$, (B) IL-6, (C) MCP-I, and (D) TNF- $\alpha$ in colon tissues of mice from different groups. Eight mice per group; ${ }^{\#} P<0.05$, compared with DSS group. ${ }^{\$} P<0.05$, compared with selenocysteine group. ${ }^{\circledR} P<0.05$, compared with selenocystine group. Data are expressed as mean \pm SEM. 
A

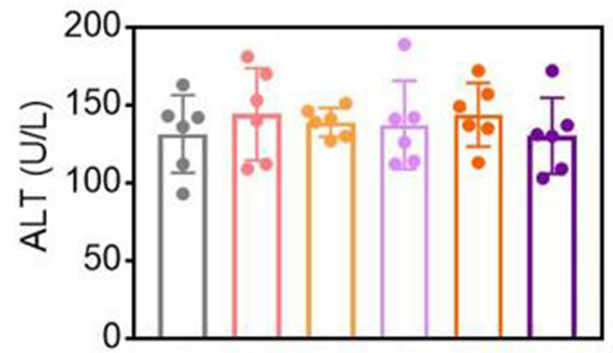

C

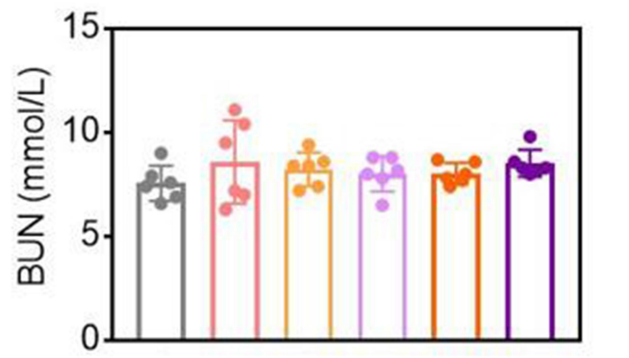

B

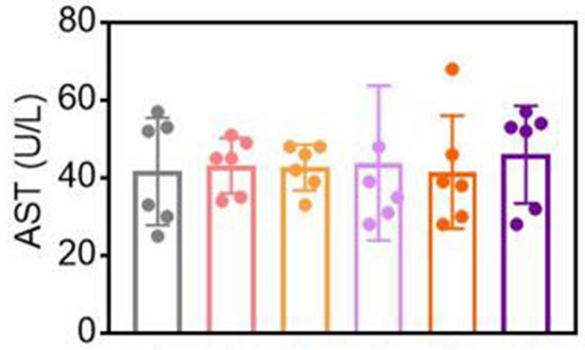

D

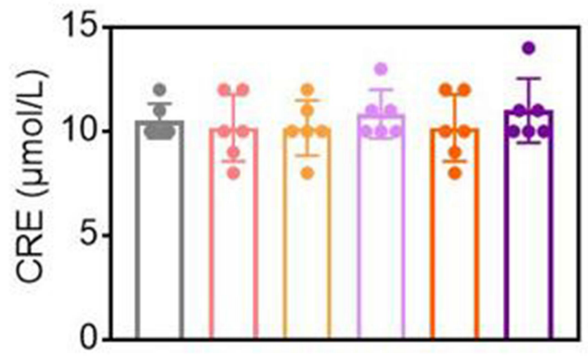

Control $=$ DSS $\square$ Selenocysteine+DSS $\square$ Selenocystine+DSS

Sodium selenite+DSS

n-acetylcysteine+DSS

Figure 6 Effects of selenium-containing amino acids on biochemical test parameters in DSS-induced IBD in mice. Serum levels of (A) ALT, (B) AST, (C) BUN, and (D) CRE in different groups. Differences were assessed via one-way analysis of variance (ANOVA) with Tukey's multiple comparison tests. Six mice per group; Data are expressed as mean \pm SEM.

that there were minimal side effects of the seleniumcontaining amino acids.

\section{Discussion}

The major novel findings of this study are summarized as follows: (1) Selenocysteine and selenocystine significantly attenuated the main symptoms of IBD, as evidenced by stable body weight, a decrease in DAI scores, and an increase in colon length in the DSS-challenged mice. (2) Selenocysteine and selenocystine significantly ameliorated DSS-induced oxidative stress, as evidenced by decreased levels of ROS and MDA activity and increased SOD and GPx activity in the mice. (3) DSS-challenged mice treated with selenocysteine or selenocystine showed significantly lower levels of pro-inflammatory cytokines (IL-1, MCP-1, IL-6, and TNF- $\alpha$ ) compared to the control mice. (4) There were minimal side effects of the selenium-containing amino acids in the DSS-challenged mice. These findings suggest that selenocysteine and selenocystine can ameliorate DSS-induced IBD via attenuating oxidative stress and intestinal inflammation. Therefore, these selenium-containing amino acids have potential as novel and safe agents in preventing and treating IBD.

Although the etiology and pathogenic mechanisms underlying IBD are not fully understood, it has been thought that oxidative stress and abnormalities in cellular immunity and autoimmunity play important roles in IBD pathogenesis. ${ }^{20,21}$ Previous studies have shown that selenium-containing agents significantly scavenge ROS and ameliorate oxidative stress, ${ }^{22}$ and that these compounds also act on the immune system to promote the proliferation of lymphocytes, induce expression of cytokine receptors, and enhance the activity of both natural killer and cytotoxic cells. ${ }^{23}$ In this study, we found that selenocysteine and selenocystine significantly attenuated oxidative stress and intestinal inflammation in a mouse model of DSSinduced IBD, suggesting significant therapeutic benefits of these selenium-containing agents in mice.

The oxidative system mainly includes excessive ROS that can induce oxidative stress, trigger lipid peroxidation, attack cell membrane lipids, and cause colonic mucosal damage. ${ }^{24,25}$ In response to injury, neutrophils, monocytes, eosinophils, and macrophages from the blood are activated to produce free radicals and ROS, which act as mediators that promote the inflammatory response. ${ }^{26}$ They also have cytotoxic effects. This killing effect on target cells is non-specific, and can therefore damage the intestinal mucosa while killing pathogens. The development and progression of IBD are also closely related to lipid peroxidation induced by oxygen free radicals. ${ }^{27}$ SOD is an important peroxide decomposing enzyme, and MDA is the 


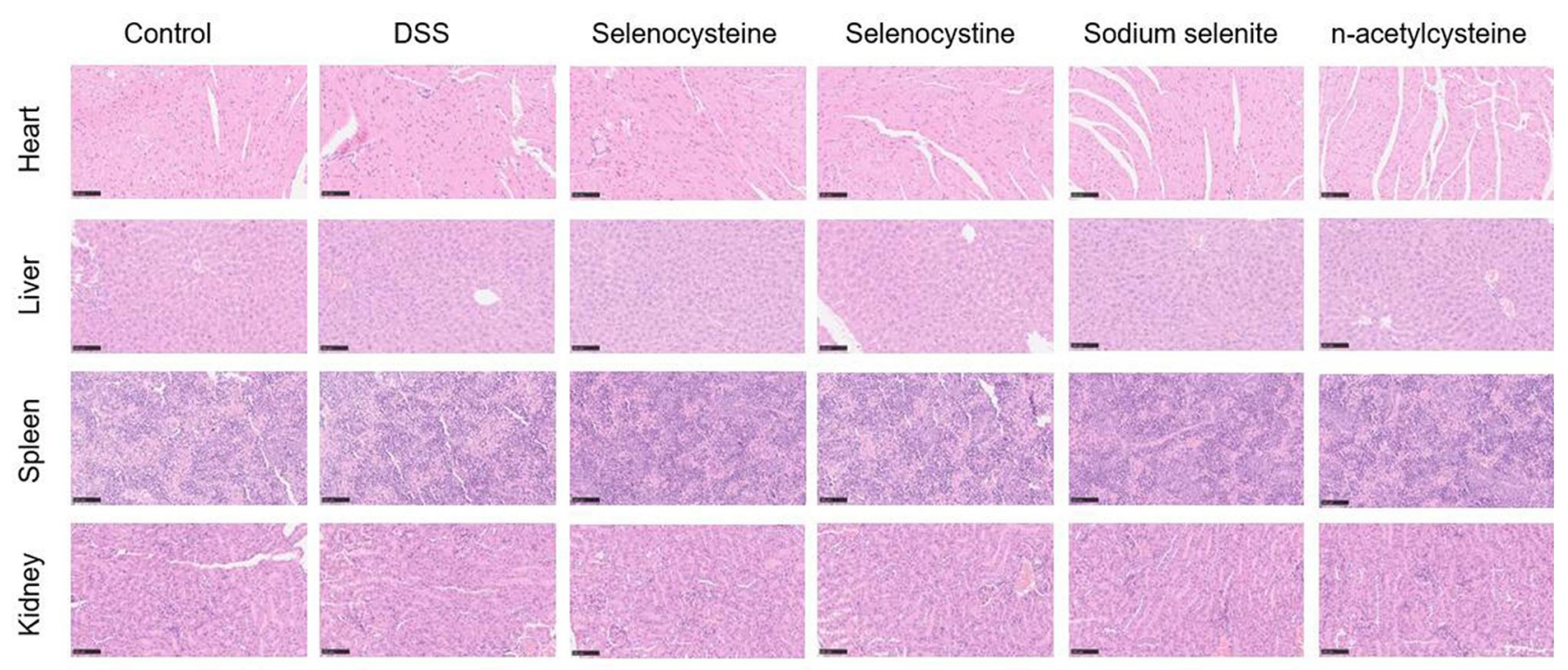

Figure 7 Histological examination of the safety of selenium-containing amino acids. Lung, kidney, heart, liver, and spleen tissues were collected, H\&E stained, and analyzed for the safety profile of selenium-containing amino acids. Scale bar is $100 \mu \mathrm{m}$.

final product of lipid peroxidation. ${ }^{28}$ It has been shown that SOD can inhibit lipid peroxidation in intestinal tissues and stabilize the cell membrane via reducing levels of oxygen free radicals. ${ }^{29}$ In fact, SOD activity can partly reflect cell membrane function and the body's anti-inflammatory response. The level of MDA largely relies on the toxic effects of reactive oxygen radicals. Excessive oxygen radicals cause lipid peroxidation of unsaturated fatty acids on biofilm and increase peroxides in tissues, thereby enhancing the secretion of peroxide decomposition products, such as MDA. ${ }^{30}$ This leads to the destruction of the cell membrane structure, an increase in the release of inflammatory cytokines, and aggravated tissue damage. MDA is an important indicator of the oxidative stress response that can reflect the degree of lipid peroxides and indirectly assess the degree of cell and tissue damage. The anti-oxidant system mainly includes antioxidants (eg vitamin $\mathrm{C}$ and vitamin $\mathrm{E}$ ) and anti-oxidant enzymes (eg GPx and SOD). ${ }^{31}$ The anti-oxidant system can scavenge oxygen free radicals, prevent lipid peroxidation, and maintain cell membrane stability. ${ }^{32}$ For example, SOD can decompose active oxygen radicals into water and oxygen molecules, quench superoxide ions, and protect cells from the cytotoxic effects of active oxygen radicals. ${ }^{33}$ In this study, MDA, ROS, and GPx levels in the colon tissues of mice in the $\mathrm{NAC}$ and selenium and selenium protein treatment groups were significantly reduced, while the SOD level was significantly increased, suggesting that NAC and selenium- containing amino acids can inhibit inflammation by regulating the redox system.

Cytokines are mainly produced by activated $\mathrm{T}$ helper $(\mathrm{Th}) 1$ and Th2 cells, ${ }^{34}$ some of which are formed by mononuclear macrophages and plasma cells. Cytokines are mainly involved in immune and inflammatory reactions. ${ }^{35}$ For instance, IL-1 has been shown to be involved in the early stage of inflammation, and it can also stimulate the production of IL-2 and IL- $6{ }^{36}$ MCP-1 is mainly produced by Th2 cells and induces the growth and differentiation of Th2 cells and the proliferation of secreted B cells. ${ }^{37}$ MCP-1 is considered a key cytokine that stimulates the Th2 immune response. ${ }^{38}$ IL-6 is a key inflammatory response factor, and its level is elevated in IBD. ${ }^{39}$ TNF$\alpha$ can induce the proliferation and activation of lymphocytes and macrophages in the colon, and strengthen the cytotoxic effect of cytotoxic T cells, leading to intestinal mucosal microcirculation disorders in IBD patients. ${ }^{40} \mathrm{TNF}-\alpha$ is an important regulatory factor in IBD and a major factor that regulates the proliferation and apoptosis of intestinal epithelial cells. ${ }^{41}$ In this study, we found that serum levels of anti-inflammatory factors IL-1, MCP-1, IL-6, and TNF- $\alpha$, which are crucial in the progress of intestinal mucosal inflammation in IBD, were significantly reduced in response to selenium-containing amino acids. There is a possibility that selenium-containing amino acids affect the release of inflammatory factors, thereby regulating the immune response via exerting their antiinflammatory properties. 
Selenium, an active oxygen free radical scavenger and catalyst, has been shown to remove lipid peroxidation free radicals and hydroxyl free radicals, thereby protecting cell membranes. ${ }^{42}$ Selenium is also an essential component of GPx that regulates its activity. ${ }^{43}$ Selenium-containing proteins are a class of natural occurring organic selenium with two different metabolic pathways. Selenium-containing proteins enter the inorganic selenium metabolism pool as a precursor for the synthesis of various selenium-containing amino acids and proteins, embedded in various proteins in the form of selenocysteine. In addition, selenium-containing proteins enter the methylated selenium metabolic pool to generate methyl selenol as a source of methylated selenium. It is evident that organic selenium has greater effects on enhancing the activity of GPx compared with inorganic selenium. ${ }^{44}$ In our study, selenium-containing amino acids significantly increased the SOD activity and the effects were better than sodium selenite alone. The selenium-containing amino acids led to better anti-oxidant and anti-inflammatory effects in mice compared to sodium selenite. These findings are promising, and future in-depth studies are needed to elucidate the molecular mechanisms underlying the anti-inflammatory effects of selenium-containing amino acids.

Our findings support the proposed mechanisms of selenium-containing amino acids and their therapeutic effects on IBD. The gut barrier is essential for host defense and its dysfunction has been associated with colitis. Under the condition of selenium deficiency, intestinal permeability is decreased, while pro-inflammatory cytokines (eg TNF- $\alpha$, IL13) are increased. As such, a Se-deficient diet is associated with increased sensitivity to DSS-induced IBD in mice. Notably, dietary selenium supplementation can modulate the immune response, alter the profile of inflammatory cytokines, and promote the resolution of inflammation, potentially conferring protection in IBD.

In summary, this study provides the first scientific evidence, to the best of our knowledge, that selenocysteine and selenocystine ameliorate IBD via attenuating oxidative stress and intestinal inflammation in mice. These findings may have clinical implications in that selenium-containing amino acids may be potentially novel and safe agents in the prevention or treatment of IBD.

\section{Data Sharing Statement}

All figures adopted to support the findings of the present study are included in the article.

\section{Acknowledgments}

This work was financially supported by the National Natural Science Foundation of China (No. 81870380).

\section{Author Contributions}

All authors have made significant intellectual contributions to this study, including in the study conception, design, execution, acquisition of data, and/or data analysis and interpretation. All authors have drafted, revised or critically reviewed the article. All authors have given final approval of the manuscript, agreed on the journal to which the article has been submitted, and agree to be accountable for all aspects of this study.

\section{Disclosure}

The authors declare that there is no conflict of interest.

\section{References}

1. Neurath MF. Targeting immune cell circuits and trafficking in inflammatory bowel disease. Nat Immunol. 2019;20:970-979. doi:10.1038/ s41590-019-0415-0

2. Kaplan GG, Ng SC. Understanding and Preventing the Global Increase of Inflammatory Bowel Disease. Gastroenterology. 2017;152:313-21 e2. doi:10.1053/j.gastro.2016.10.020

3. Michielan A, D'Inca R. Intestinal Permeability in Inflammatory Bowel Disease: pathogenesis, Clinical Evaluation, and Therapy of Leaky Gut. Mediators Inflamm. 2015;2015:628157. doi:10.1155/2015/628157

4. Kinchen J, Chen HH, Parikh K, et al. Structural Remodeling of the Human Colonic Mesenchyme in Inflammatory Bowel Disease. Cell. 2018;175:372-86 e17. doi:10.1016/j.cell.2018.08.067

5. Se B, As S, Lowenberg M. Clinical Pharmacokinetic and Pharmacodynamic Considerations in the Treatment of Ulcerative Colitis. Clin Pharmacokinet. 2019;58:15-37. doi:10.1007/s40262-018-0676-z

6. Melo FJ, Magina S. Clinical management of Anti-TNF-alpha-induced psoriasis or psoriasiform lesions in inflammatory bowel disease patients: a systematic review. Int J Dermatol. 2018;57:1521-1532. doi:10.1111/ ijd. 14072

7. Nikkhah-Bodaghi M, Maleki I, Agah S, Hekmatdoost A. Zingiber officinale and oxidative stress in patients with ulcerative colitis: A randomized, placebo-controlled, clinical trial. Complement Ther Med. 2019;43:1-6. doi:10.1016/j.ctim.2018.12.021

8. Oh SJ, Seo Y, Ahn JS, et al. Echinochrome A Reduces Colitis in Mice and Induces In Vitro Generation of Regulatory Immune Cells. Mar Drugs. 2019;17. doi:10.3390/md17110622

9. Neubauer K, Kempinski R, Matusiewicz M, Bednarz-Misa I. Nonenzymatic Serum Antioxidant Capacity in IBD and Its Association with the Severity of Bowel Inflammation and Corticosteroids Treatment. Medicina. 2019;55. doi:10.3390/medicina55040088

10. Valea A, Georgescu CE. Selenoproteins in human body: focus on thyroid pathophysiology. Hormones. 2018;17:183-196. doi:10.1007/ s42000-018-0033-5

11. Shao D, Li M, Wang Z. Bioinspired Diselenide-Bridged Mesoporous Silica Nanoparticles for Dual-Responsive Protein Delivery. $A d v$ Mater. 2018;e1801198. doi:10.1002/adma.201801198

12. Avery JC, Hoffmann PR. Selenium, Selenoproteins, and Immunity. Nutrients. 2018;10. doi:10.3390/nu10091203

13. Mehdi Y, Hornick JL, Istasse L, Dufrasne I. Selenium in the environment, metabolism and involvement in body functions. Molecules. 2013;18:3292-3311. doi:10.3390/molecules 18033292 
14. Andoh A, Hirashima $M$, Maeda $H$, et al. Serum selenoprotein-P levels in patients with inflammatory bowel disease. Nutrition. 2005;21:574-579. doi:10.1016/j.nut.2004.08.025

15. Kaushal N, Kudva AK, Patterson AD, et al. Crucial role of macrophage selenoproteins in experimental colitis. $J$ Immunol. 2014;193:3683-3692. doi:10.4049/jimmunol.1400347

16. Hiller F, Oldorff L, Besselt K, Kipp AP. Differential acute effects of selenomethionine and sodium selenite on the severity of colitis. Nutrients. 2015;7:2687-2706. doi:10.3390/nu7042687

17. Wang J, Zhao B, Wang X, Yao J, Zhang J. Synthesis of selenium-containing polysaccharides and evaluation of antioxidant activity in vitro. Int $J$ Biol Macromol. 2012;51:987-991. doi:10.1016/j.ijbiomac.2012.08.011

18. Misra S, Boylan M, Selvam A, Spallholz JE, Bjornstedt M. Redoxactive selenium compounds-from toxicity and cell death to cancer treatment. Nutrients. 2015;7:3536-3556. doi:10.3390/nu7053536

19. Rahmanto AS, Davies MJ. Selenium-containing amino acids as direct and indirect antioxidants. IUBMB Life. 2012;64:863-871. doi:10.1002/iub.1084

20. Roque Ramos L, DiMaio CJ, Sachar DB, Atreja A, Colombel JF, Torres J. Autoimmune pancreatitis and inflammatory bowel disease: case series and review of the literature. Dig Liver Dis. 2016;48:893-898. doi:10.1016/j.dld.2016.05.008

21. Almousa AA, Meurens F, Krol ES, Alcorn J. Linoorbitides and enterolactone mitigate inflammation-induced oxidative stress and loss of intestinal epithelial barrier integrity. Int Immunopharmacol. 2018;64:42-51. doi:10.1016/j.intimp.2018.08.012

22. Ertilav K, Naziroglu M, Ataizi ZS, Braidy N. Selenium Enhances the Apoptotic Efficacy of Docetaxel Through Activation of TRPM2 Channel in DBTRG Glioblastoma Cells. Neurotox Res. 2019;35:797-808. doi:10.1007/s12640-019-0009-5

23. Das K, Dupont A, De Pauw-gillet MC, Debier C, Siebert U. Absence of selenium protection against methylmercury toxicity in harbour seal leucocytes in vitro. Mar Pollut Bull. 2016;108:70-76. doi:10.1016/j. marpolbul.2016.04.060

24. Dong Y, Hou Q, Lei J, Wolf PG, Ayansola H, Zhang B. Quercetin Alleviates Intestinal Oxidative Damage Induced by $\mathrm{H} 2 \mathrm{O} 2$ via Modulation of GSH: in Vitro Screening and In Vivo Evaluation in a Colitis Model of Mice. ACS Omega. 2020;5:8334-8346. doi:10.1021/acsomega.0c00804

25. Den Hartog G, Chattopadhyay R, Ablack A, et al. Regulation of Rac1 and Reactive Oxygen Species Production in Response to Infection of Gastrointestinal Epithelia. PLoS Pathog. 2016;12(1):e1005382. doi:10.1371/journal.ppat.1005382

26. Poehlmann A, Reissig K, Just A, et al. Non-apoptotic function of caspases in a cellular model of hydrogen peroxide-associated colitis. J Cell Mol Med. 2013;17(7):901-913. doi:10.1111/jcmm.12079

27. Dudzinska E, Gryzinska M, Ognik K, Gil-Kulik P, Kocki J. Oxidative Stress and Effect of Treatment on the Oxidation Product Decomposition Processes in IBD. Oxid Med Cell Longev. 2018;2018:7918261. doi:10.1155/2018/7918261

28. Mrowicka M, Mrowicki J, Mik M, Ł D, Dziki A, Majsterek I. Assessment of DNA damage profile and oxidative/antioxidative biomarker level in patients with inflammatory bowel disease. Pol Przegl Chir. 2020;92(5):8-15. doi:10.5604/01.3001.0014.1548

29. Hou CL, Zhang J, Liu XT, Liu H, Zeng XF, Qiao SY. Superoxide dismutase recombinant Lactobacillus fermentum ameliorates intestinal oxidative stress through inhibiting NF-kappaB activation in a trinitrobenzene sulphonic acid-induced colitis mouse model. J Appl Microbiol. 2014;116(6):1621-1631. doi:10.1111/jam.12461
30. Aki HS, Fujita M, Yamashita S, et al. Elevation of jugular venous superoxide anion radical is associated with early inflammation, oxidative stress, and endothelial injury in forebrain ischemia-reperfusion rats. Brain Res. 2009;1292:180-190. doi:10.1016/j. brainres.2009.07.054

31. Diaz de Barboza G, Guizzardi S, Moine L. Oxidative stress, antioxidants and intestinal calcium absorption. World J Gastroenterol. 2017;23(16):2841-2853. doi:10.3748/wjg.v23.i16.2841

32. Pisoschi AM, Pop A. The role of antioxidants in the chemistry of oxidative stress: A review. Eur J Med Chem. 2015;97:55-74. doi:10.1016/j.ejmech.2015.04.040

33. Offer T, Samuni A. Nitroxides inhibit peroxyl radical-mediated DNA scission and enzyme inactivation. Free Radic Biol Med. 2002;32 (9):872-881. doi:10.1016/s0891-5849(02)00750-5

34. Schulke S. Induction of Interleukin-10 Producing Dendritic Cells As a Tool to Suppress Allergen-Specific T Helper 2 Responses. Front Immunol. 2018;9:455. doi:10.3389/fimmu.2018.00455

35. Chernyavsky AI, Arredondo J, Skok M, Grando SA. Auto/paracrine control of inflammatory cytokines by acetylcholine in macrophage-like U937 cells through nicotinic receptors. Int Immunopharmacol. 2010;10:308-315. doi:10.1016/j.intimp.2009.12.001

36. Lawlor KE, Wong PK, Campbell IK, van Rooijen N, Wicks IP. Acute CD4+ T lymphocyte-dependent interleukin-1-driven arthritis selectively requires interleukin- 2 and interleukin- 4 , joint macrophages, granulocyte-macrophage colony-stimulating factor, interleukin-6, and leukemia inhibitory factor. Arthritis Rheum. 2005;52:3749-3754. doi:10.1002/art.21495

37. Cortes-Selva D, Ready A, Gibbs L, Rajwa B, Fairfax KCIL-4. promotes stromal cell expansion and is critical for development of a type-2, but not a type 1 immune response. Eur J Immunol. 2019;49:428-442. doi:10.1002/eji.201847789

38. Chu DK, Mohammed-Ali Z, Jimenez-Saiz R, et al. T helper cell IL-4 drives intestinal Th2 priming to oral peanut antigen, under the control of OX40L and independent of innate-like lymphocytes. Mucosal Immunol. 2014;7:1395-1404. doi:10.1038/mi.2014.29

39. Hunter CA, Jones SAIL-6. as a keystone cytokine in health and disease. Nat Immunol. 2015;16:448-457. doi:10.1038/ni.3153

40. Kawamoto A, Nagata S, Anzai S, et al. D is Upregulated by Synergy of Notch Signalling and TNF-alpha in the Inflamed Intestinal Epithelia of IBD Patients. J Crohns Colitis. 2019;13:495-509. doi:10.1093/ecco-jcc/jjy180

41. Tang R, Yang G, Zhang S, Wu C, Chen M. Opposite effects of interferon regulatory factor 1 and osteopontin on the apoptosis of epithelial cells induced by TNF-alpha in inflammatory bowel disease. Inflamm Bowel Dis. 2014;20:1950-1961. doi:10.1097/ MIB.0000000000000192

42. Tarrahi R, Khataee A, Movafeghi A, Rezanejad F, Gohari G. Toxicological implications of selenium nanoparticles with different coatings along with $\mathrm{Se}(4+)$ on Lemna minor. Chemosphere. 2017;181:655-665. doi:10.1016/j.chemosphere.2017.04.142

43. Rusetskaya NY, Fedotov IV, Koftina VA, Borodulin VB. [Selenium compounds in redox regulation of inflammation and apoptosis]. Biomed Khim. 2019;65:165-179. doi:10.18097/PBMC20196503165

44. Ilham I, Siddik MA, Fotedar R. Effects of Organic Selenium Supplementation on Growth, Accumulation, Haematology and Histopathology of Juvenile Barramundi (Lates calcarifer) Fed High Soybean Meal Diets. Biol Trace Elem Res. 2016;174:436-447. doi:10.1007/s12011-016-0708-1 


\section{Publish your work in this journal}

The Journal of Inflammation Research is an international, peerreviewed open-access journal that welcomes laboratory and clinical findings on the molecular basis, cell biology and pharmacology of inflammation including original research, reviews, symposium reports, hypothesis formation and commentaries on: acute/chronic inflammation; mediators of inflammation; cellular processes; molecular mechanisms; pharmacology and novel anti-inflammatory drugs; clinical conditions involving inflammation. The manuscript management system is completely online and includes a very quick and fair peerreview system. Visit http://www.dovepress.com/testimonials.php to read real quotes from published authors.

Submit your manuscript here: https://www.dovepress.com/journal-of-inflammation-research-journal 